\title{
BEDAQUILINE: A long but much fruitful wait since seventies
}

\author{
Anjan Khadka ${ }^{1}$. \\ ${ }^{1}$ Department of Pharmacology, Armed Forces Medical College, Pune, India.
}

\begin{abstract}
Introduction : Tuberculosis (TB) is a major health problem in developing countries one of the leading cause of deaths due to infectious disease in the world. Drug treatment had being successful with anti-tubercular drugs available, but Multidrug-resistant tuberculosis (MDR-TB) and extensively drug-resistant TB (XDR-TB) are serious forms of TB which have emerged as real concern. Bedaquiline (TMC-207 or R207910), is a new anti TB agent been approved as a part of drug regimen therapy in pulmonary MDR-TB. Bedaquiline acts on energy metabolism of the bacteria. It acts as a bactericidal drug. There are two important black box warnings for this drug, firstly prolongation of QT interval and secondly increased risk of death with bedaquiline as compared to placebo treatment.Fast track approval for bedaquiline was based on two phase 2 trials. The FDA has approved bedaquiline on Dec 2012, for the treatment of MDR-TB in adults ( $>18$ years) as part of combination therapy under the fast track designation, priority review and orphan-product designation based on two Phase II trials. Careful use of this drug along with monitoring of the potential adverse effects and drug interactions becomes very important, as this new anti-tubercular drug has being approved after forty years, since last anti tubercular drug was approved.
\end{abstract}

Key Messages : Tuberculosis is a global health problem. Effective TB treatment is difficult mostly due to the unusual structure and chemical composition of the mycobacterial cell wall. Active TB disease is best treated with combinations of several drugs like isoniazid, rifampicin, pyrazinamide, ethambutol, streptomycin, etc. There is increased emergence of multiple drug-resistant tuberculosis (MDR-TB). Bedaquiline is a diarylquinoline antituberculosis drug with novel mechanism of action and potent activity against drug-sensitive and drug-resistant TB. It is the first new medicine to fight TB in more than forty years and is specifically approved to treat multidrug-resistant tuberculosis.

Keywords: bedaquiline; mycobacteria; tuberculosis.

\section{INTRODUCTION}

Tuberculosis (TB) is a one of the leading cause of deaths due to infectious disease in the world. ${ }^{[1]}$ It is caused by acid fast bacilli, Mycobacterium tuberculosis. In 1993 World Health Organization (WHO) introduced "directly observed therapy short-course" (DOTS) as a global step to control TB. ${ }^{[1]}$

Multidrug resistant TB (MDR-TB) and extensively drug-resistant TB (XDR-TB) are serious forms of TB which have emerged as real concern. ${ }^{[2]}$ MDR-TB are resistant to at least two most powerful first-line antiTB drugs, isoniazid and rifampicin and XDR-TB are resistant to isoniazid and rifampicin, in addition to any fluoroquinolone, and to at least one of the three following injectable drugs: capreomycin, kanamycin or amikacin. ${ }^{[2]}$

WHO has reported that about $3.7 \%$ of newly diagnosed TB patients globally have MDR-TB strains, whereas, in previously treated TB patients it was around $20 \%$. ${ }^{[3,4]}$ Present challenges faced with MDR-TB treatment are cost factor, effectiveness, prolonged regimen and

Correspondence:

Anjan Khadka.

Department of Pharmacology, Armed Force Medical

College, Pune.

Email:anjankhadka14@yahoo.com 
poor tolerance by the patients. ${ }^{[4]}$ Need for a new antitubercular drug became a need for the hour. Last drug approved was back in seventies around four decades back. ${ }^{[5]}$

Bedaquiline (TMC-207 or R207910), is a new anti TB agent been approved as a part of drug regimen therapy in pulmonary MDR-TB. ${ }^{[5]}$ It is effective TB even for those strains that are resistant to streptomycin, pyrazinamide, ethambutol and moxifloxacin. ${ }^{[5,6]}$ It has bactericidal and sterilizing activity against $\mathrm{M}$. tuberculosis and other mycobacterial species. It is found to active within macrophages, and is a promising agent in shortening the duration of anti- TB treatment. [6]

Pharmacokinetics: The maximum plasma concentration (Cmax) is reached after $5 \mathrm{~h}$ with a half-life of 173 hours. Its highly plasma protein bound $(99 \%)^{[7,8]}$ Its bioavailability is increased with food. ${ }^{[8]}$ It is metabolized to an active metabolite $\mathrm{N}$-monodesmethyl in liver, mainly by CYP3A4. This active metabolite has lesser bactericidal activity as compared to the parent drug. ${ }^{[8]}$

Mechanism of action and antimicrobial properties: Bedaquiline acts on energy metabolism of the bacteria.

${ }^{[9]}$ It inhibits adenosine 5'-triphosphate (ATP) synthase a crucial enzyme for Mycobacterium tuberculosis growth. ${ }^{[7,9]}$ It inhibits proton pump activity of the enzyme by acting on subunit $\mathrm{C}$ of the enzyme, leading to bacterial death, hence it acts as a bactericidal drug. $[7,9]$

Bedaquiline has minimal inhibitory concentration (MIC) of 0.03-0.12 mg/L, for both drug susceptible and resistant strains of mycobacterium. ${ }^{[6,7,9]}$ Even dormant bacterias are killed. This additional cidal property makes it more superior to other first line anti TB drugs. ${ }^{[7,9]}$

\section{ADVERSE EVENTS, PRECAUTION AND CONTRAINDICATION}

Common adverse effects are nausea and diarrhoea, arthralgia, pain in extremities, hyperuricemia, rash, and somnolence. ${ }^{[8,9]}$ There are two important black box warnings for this drug, firstly prolongation of QT interval and secondly increased risk of death with bedaquiline as compared to placebo treatment. Therefore it should be only used when other regimens are not effective or tolerated. ${ }^{[8]}$ In all patients being put on bedaquiline, ECG should be done before starting treatment and at least 2, 12, and 24 weeks after starting therapy. ${ }^{[8,10]}$ Baseline potassium, calcium, and magnesium levels should be monitored. ${ }^{[8,10]}$ It is contraindicated in patient less than 18 years of age group and with history of torsade de pointes or congenital long QT syndrome. ${ }^{[8,9]}$ It is category B, so should be used cautiously in pregnancy as data in humans are lacking. ${ }^{[8,10]}$ It is not known whether bedaquiline or its metabolites are excreted in human milk. ${ }^{[8,10]}$

\section{DRUG INTERACTIONS}

Bedaquiline is metabolized by CYP3A4. Concurrent use of strong CYP3A4 inducers (e.g., rifampin, rifapentine, rifabutin) may reduce the effect and cause treatment failure, therefore, should be avoided. ${ }^{[9,10]}$ Co administration with CYP3A4 inhibitors e.g. ketoconazole, ciprofloxacin and other quinolones may increase systemic concentrations and result in increased adverse effects and hence, should be avoided. ${ }^{[10]}$

\section{CLINICAL TRIALS}

In an early bactericidal study, seventy five previously untreated were positive or pulmonary TB were randomized into three arms. ${ }^{[7,10]}$ First one was given oral bedaquiline ( $25 \mathrm{mg}, 100 \mathrm{mg}$, or $400 \mathrm{mg}$ ), second group was given $600 \mathrm{mg}$ rifampin and third group was given $300 \mathrm{mg}$ isoniazid for 7 days. ${ }^{[7,10]}$ The bactericidal activity was expressed as the log (10) decrease in $\mathrm{CFU} / \mathrm{ml}$ sputum/day. Significant cidal activity was seen with bedaquiline and was similar to other two drugs. Bedaquiline demonstrated delayed onset of bactericidal activity (from day 4 ). ${ }^{[10]}$

Fast Track for bedaquiline was based on placebocontrolled, double blind, randomized trial, done on multi-drug resistant pulmonary mycobacterium tb positive patients. They were divided into two arms, first one $(n=79)$ was given bedaquiline and other standard regimen having ethionamide, kanamycin, pyrazinamide, ofloxacin, and cycloserine/terizidone or available alternative, whereas second $\operatorname{arm}(\mathrm{n}=81)$ was given placebo with standard regimen. ${ }^{[5,6]}$ bedaquiline was dosed as $400 \mathrm{mg}$ once daily for the first 2 weeks and as $200 \mathrm{mg} 3$ times per week for the following 22 weeks. ${ }^{[5,6]}$ after 24 week treatment standard regimen continued for 18 to 24 months, or for at least 12 months after first negative culture was detected. ${ }^{[5,6]}$ bedaquiline significantly decreased the time to sputum culture conversion. ${ }^{[6]}$ 


\section{PRESENT STATUS}

The FDA has approved bedaquiline on Dec 2012, for the treatment of MDR-TB in adults ( $>18$ years) as part of combination therapy under the fast track designation, priority review and orphan-product designation based on two Phase II trials. ${ }^{[6,7]}$ The dose is $400 \mathrm{mg}$ ( four tablets of $100 \mathrm{mg}$ ) once daily for first two week and then $200 \mathrm{mg} 3$ times a week (with at least 48 hours between doses) with total dose of 600 mg per week. It should only be administered as part of amdr-tb regimen under dots. The total duration of treatment is 24 weeks. ${ }^{[7,8,9]}$ the safety and efficacy of bedaquiline in children and adolescents $<18$ years of age have not been studied. ${ }^{[5,6,8]}$

\section{CONCLUSIONS}

Bedaquiline has come to market as new anti TB agent after 40 years. It has a novel mechanism of action and potent activity against drug-sensitive and drug-resistant TB. Though this drug has limitations e.g. adverse effects like prolongation of QT interval, nausea and drug interaction with CYP3A4 inducers and inhibitors, it is an attractive option for MDR and XDR- TB since it shortens the duration of anti- TB therapy.

\section{REFERENCES}

1. Giri PA, Deshpande JD, Phalke DB. Prevalence of Pulmonary Tuberculosis among HIV Positive Patients Attending Antiretroviral Therapy. Clinic.N Am J Med Sci. 2013 Jun; 5(6):367-70. doi: 10.4103/1947-2714.114169. http://dx.doi. org/10.4103/1947-2714.114169

2. Podewils LJ, Gler MT, Quelapio MI, Chen MP. Patterns of Treatment Interruption among Patients with Multidrug-Resistant TB (MDR TB) and Association with Interim and Final Treatment Outcomes. PLoS One. 2013 Jul 29; 8(7):e70064. http://dx.doi.org/10.1371/journal.pone.0070064

3. Singh J, Sankar MM, Kumar S, Gopinath K, Singh N, Mani K, Singh S. Incidence and Prevalence of Tuberculosis among Household Contacts of Pulmonary Tuberculosis Patients in a Peri-Urban
Population of South Delhi, India. PLoS One. 2013 Jul 26; 8(7):e69730. http://dx.doi.org/10.1371/ journal.pone.0069730

4. Voelker R. MDR-TB has new drug foe after fasttrack approval. JAMA. 2013 Feb 6; 309(5):430. http://dx.doi.org/10.1001/jama.2013.94

5. Cohen J. Infectious disease. Approval of novel TB drug celebrated--with restraint. Science. 2013 Jan 11; 339(6116):130. http://dx.doi.org/10.1126/ science.339.6116.130

6. Gras J. Bedaquiline for the treatment of pulmonary, multidrug-resistant tuberculosis in adults. Drugs Today (Barc). 2013 Jun; 49(6):353-61.

7. Dooley KE, Park JG, Swindells S, Allen R, et al.Safety, tolerability, and pharmacokinetic interactions of the antituberculous agent TMC207 (bedaquiline) with efavirenz in healthy volunteers: AIDS Clinical Trials Group Study A5267. J Acquir Immune DeficSyndr. 2012 Apr 15; 59(5):455-62. http://dx.doi.org/10.1097/QAI.0b013e3182410503

8. Diacon AH, Pym A, Grobusch M, Patientia R, et al.Thediarylquinoline TMC207 for multidrugresistant tuberculosis.NEngl J Med. 2009 Jun 4; 360(23):2397-405 http://dx.doi.org/10.1056/ NEJMoa0808427

9. Avorn J. Approval of a tuberculosis drug based on a paradoxical surrogate measure. JAMA. $2013 \mathrm{Apr}$ 3; 309(13):1349-50. http://dx.doi.org/10.1001/ jama.2013.623

10. Diacon AH, Donald PR, Pym A, Grobusch M, et al. Randomized pilot trial of eight weeks of bedaquiline (TMC207) treatment for multidrugresistant tuberculosis: long-term outcome, tolerability, and effect on emergence of drug resistance. Antimicrob Agents Chemother. 2012 Jun; 56(6):3271-6. http://dx.doi.org/10.1128/ AAC.06126-112 